\title{
Der Musterarbeitsvertrag für MPA
}

In Zusammenarbeit mit den MPA-Verbänden werden auf der FMH-Homepage ein Arbeitsvertrag für Medizinische Praxisassistentinnen sowie Allgemeine Rahmenbedingungen und Erläuterungen zum Arbeitsvertrag zur Verfügung gestellt. Der Arbeitsvertrag sowie die dazugehörenden Rahmenbedingungen sind seit längerem nicht mehr überarbeitet worden. Letzte Änderungen wurden im Jahr 2009 vorgenommen. Der Arbeitsvertrag sowie die Allgemeinen Rahmenbedingungen wurden revidiert und ergänzt. Die Anpassungen und Ergänzungen haben vor allem informativen Charakter und betreffen arbeitsrechtliche Schwerpunkte. Mit Ausnahme der Bestimmungen für stillende Mütter (Inkrafttreten der Änderungen der Verordnung 1 zum Arbeitsgesetz am 1. Juni 2014) stellen die Änderungen und Ergänzungen kein neues Recht dar. Bisher verwendete Arbeitsverträge können weiterhin angewendet bleiben.

\section{Arbeitsvertrag}

Ein Musterarbeitsvertrag findet sich unter dem Pfad www.fmh.ch $\rightarrow$ Services $\rightarrow$ Medizinische Praxisassistentinnen $\rightarrow$ Arbeitsbedingungen bzw. dem Link www. fmh.ch/services/medizinische_praxisassistentin/ arbeitsbedingungen.html

\section{Änderungen im Arbeitsvertrag}

\section{ad. Ziff. 2 Arbeitszeit und Überstunden}

Wenn immer möglich, sollen die Überstunden durch Freizeit oder allfällige Mehrferien (= Ferien, welche über dem gesetzlichen Minimum von 4 Wochen liegen) gleicher Dauer ausgeglichen werden.

\section{ad. Ziff. 5.3 Gesundheitsschutz}

Da sich die Empfehlungen des Bundesamtes für Gesundheit betreffend Impfempfehlungen laufend ändern können, soll neu auf die aktuell gültigen Empfehlungen verwiesen werden.

\section{ad. Ziff. 6.7 Schwangere Frauen und stillende Mütter}

Zu ergänzen ist Ziffer 6.7 damit, dass stillenden Müttern die erforderliche Zeit zum Stillen freizugeben ist.
Weitere Informationen finden sich in den Rahmenbedingungen.

\section{ad. Ziff. 6.9 und 8.4 (neu)}

Ein Betrieb mit gefährlichen und beschwerlichen Arbeiten hat grundsätzlich eine Risikobeurteilung vorzunehmen. Gefährliche und beschwerliche Arbeiten sollen - wenn möglich - durch geeignete Schutzmassnahmen ausgeschaltet oder der MPA soll eine Ersatzarbeit angeboten werden. Können schwangere Frauen und stillende Mütter bestimmte Arbeiten nicht verrichten, was ein Beschäftigungsverbot zur Folge hat, haben diese Anspruch auf 80\% des Lohnes. Im Gegensatz zur Arbeitsunfähigkeit, bei welcher die Krankentaggeldversicherung die Taggelder entrichtet, ist es beim Beschäftigungsverbot der Arbeitgeber, welcher die Lohnfortzahlung schuldet.

\section{ad. Ziff. 7.1 Ferien und Urlaubstage}

Der Arbeitgeber hat der MPA mit Familienpflichten die zur Betreuung kranker Kinder erforderliche Zeit im Umfang bis zu drei Tagen freizugeben. Der Arbeitgeber kann in diesem Fall von der MPA verlangen, dass sie ein ärztliches Zeugnis vorzulegen hat.

\section{ad. Ziff. 7.2 Berufliche Fortbildung}

Zur Förderung der MPA sollen dieser neu jährlich 3 anstatt 2 - bezahlte, nicht an Ferien oder Ruhetage anrechenbare Urlaubstage zum Besuch von beruflichen Fortbildungsveranstaltungen zustehen.

\section{ad. Ziff. 8.1 Verhinderung an der Arbeitsleistung;} Lohnfortzahlung, Versicherungen

Der Lohnfortzahlungsanspruch soll der MPA nicht nur bei einem «krankhaften» Schwangerschaftsverlauf zustehen. Um sicherzustellen, dass der Lohnfortzahlungsanspruch nicht an dem fehlenden Krankheitswert von Schwangerschaftsbeschwerden scheitern kann, wird der Satz eingefügt, dass bei Arbeitsverhinderungen aus gesundheitlichen Gründen infolge von Schwangerschaft (sog. Risikoschwangerschaft) der Arbeitgeber zur Lohnfortzahlung in gleichem Umfang wie bei Krankheit verpflichtet ist. 


\section{Allgemeine Rahmenbedingungen und Erläuterungen zum Arbeitsvertrag}

\section{Rahmenbedingungen für Lohnverhandlungen}

Aufgrund der seit 1991 kantonal von den Ärztegesellschaften ausgearbeiteten Lohnempfehlungen für Medizinische Praxisassistentinnen werden keine gesamtschweizerischen Lohnempfehlungen mehr publiziert. Bei individuellen Lohnverhandlungen sollen für Medizinische Praxisassistentinnen die nachstehend aufgeführten Rahmenbedingungen berücksichtigt werden.

\section{Ausbildung}

- Eidg. Fähigkeitszeugnis Medizinische Praxisassistentin bzw. Diplom der Verbindung der Schweizer Ärzte (Vignette) inkl. Röntgenbewilligung.

2. Arbeitsbedingungen: Berechnungsgrundlagen

- 42-Stunden-Woche im Jahresdurchschnitt

- 4 Wochen Ferien (Medizinische Praxisassistentinnen unter 20 und ab 50 Jahren: 5 Wochen)

Nennenswerte Abweichungen von diesen Bedingungen können auf den Lohn umgerechnet werden.

\section{Regionale Gegebenheiten}

Die Löhne sollen den regionalen Gegebenheiten angepasst werden.

\section{13. Monatslohn}

Am Jahresende ist der Medizinischen Praxisassistentin ein 13. Monatslohn auszurichten. Umfasst das Arbeitsverhältnis nicht das ganze Kalenderjahr, so ist er anteilsmässig zu bezahlen.

5. Dienstalterszulagen und Reallohnerhöhungen Die Höhe einer Dienstalterszulage soll auch für 2016 ein Thema des jährlichen Qualifikationsgespräches bilden. Insbesondere sollen bei der Festlegung des Lohnes auch jede zusätzliche Verantwortung (z.B. Ausbilden von Lernenden) oder Weiterbildung (z.B. Dosisintensives Röntgen, usw.) der Angestellten berücksichtigt und der Lohn dementsprechend angepasst werden. Eine Vorlage für ein Mitarbeitergespräch finden Sie online bei der FMH, Rubrik Medizinische Praxisassistentinnen (www.fmh.ch/services/medizinische praxis assistentin.html)
6. Teuerungsausgleich (www.bfs.admin.ch) Die Teuerung soll ausgeglichen werden. Wo der Teuerungsausgleich vertraglich vereinbart ist, muss dieser auf jeden Fall gewährt werden. Im Falle einer Minusteuerung ist keine Teuerung auszugleichen. (LIKP September 2015: -1,4\%, Oktober 2015: -1.4\%, Indexbasis Dez. 2010.)

\section{Teilzeitarbeit im Monatslohn}

Bei Teilzeitarbeit beträgt der Bruttolohn (bei 42 Wochenstunden als Berechnungsgrundlage) 1/42 eines vollen Monatslohnes, multipliziert mit der Anzahl der vereinbarten Wochenarbeitsstunden.

\section{Stundenlohn}

Bei sehr geringer und gleichzeitig unregelmässiger Arbeitszeit empfiehlt sich die Ausrichtung eines Stundenlohnes. Als Stundenlohnansatz empfehlen wir 7\%o eines Monatslohnes für ein Vollzeitpensum (13. Monatslohn ist anteilsmässig darin enthalten, inkl. Feiertagsentschädigung). Zusätzlich muss auf diesem Ansatz ein Ferienanteil von 8,33\% ausgerichtet werden, der den üblichen 4 Wochen Ferien entspricht und auf jeder Lohnabrechnung separat auszuweisen ist (bei 5 Wochen Ferien 10,64\%, bei 6 Wochen Ferien 13,04\% usw.). Diese Berechnung gilt auch bei der Auszahlung von Überstunden.

\section{9. Überstunden (Ziff. 2 des Mustervertrags)}

Wenn immer möglich, sollen die Überstunden durch Freizeit oder allfällige Mehrferien (= Ferien, welche über dem gesetzlichen Minimum von 4 Wochen liegen) gleicher Dauer ausgeglichen werden. Ist dies nicht möglich, kann ausnahmsweise gewählt werden zwischen folgenden Varianten:

- Entschädigung samt einem Lohnzuschlag von 25\% für Vollzeit- und Teilzeitangestellte (Vorzugsvariante FMH / SVA nur für Vollzeitangestellte).

- Bei Teilzeitarbeit werden Überstunden, so lange sie zusammen mit dem Teilzeitpensum die betriebsübliche Arbeitszeit für eine Vollzeitangestellte nicht überschreiten, lediglich nach dem normalen Stundenansatz vergütet (Vorzugsvariante SVA ${ }^{1}$.

- Arbeitgeber und Med. Praxisassistentin können durch schriftliche Vereinbarung eine andere Regelung wählen. 
Als Stundenlohnansatz gelten 7\%o eines Monatslohns für ein Vollzeitpensum zuzüglich Ferienanteil (vgl. Ziff. 8 Stundenlohn).

Für alle vereinbarten Löhne gelten folgende Bestimmungen und Empfehlungen:

\section{Abzüge vom Bruttolohn}

- AHV, IV, EO, ALV: 6,25\% (AHV, IV, EO = 5,15\%, ALV = $1,1 \%)$

- Nichtberufsunfallversicherung (bei einer wöchentlichen Arbeitszeit von mehr als 8 Stunden): Abzug gemäss konkretem Versicherungsvertrag

- Berufliche Altersvorsorge (2. Säule BVG): Arbeitnehmeranteil (maximal und normalerweise 50\%) des altersabhängigen Beitrages gemäss Versicherungsausweis.

\section{Erläuterungen zum Mustervertrag}

\section{Arbeitszeit und Arbeitszeiterfassung} (Ziffer 2 des Mustervertrages)

Gemäss Art. 46 ArG und Art. 73 ArGV 1 besteht eine gesetzliche Aufzeichnungspflicht der Arbeitszeit durch den Arbeitgeber. Es muss für jede Arbeitnehmerin nachvollziehbar sein, wann sie gearbeitet, die Pausen bezogen und die Arbeit beendet hat. Aufgrund von diesen Angaben kann überprüft werden, ob die Arbeitsund Ruhezeitvorschriften des Arbeitsgesetzes eingehalten wurden. Der Arbeitgeber ist verpflichtet, diese Arbeitszeitaufzeichnungen während 5 Jahren aufzubewahren (Art. 73 Abs. 2 ArGV 1).

Arbeitszeiterfassung heisst nicht in jedem Fall Stempeluhr. Es gibt auch andere bewährte Möglichkeiten, die Arbeitszeiten zu erfassen (z.B. Excel-Tabelle). Wird die Arbeitszeit jedoch von der Arbeitnehmerin mit einer Excel-Tabelle selbst erfasst, empfehlen wir, dass die Zeiterfassung vom Arbeitgeber visiert wird.

Ein Excel-Tool zur Arbeitszeiterfassung finden Sie online beim SVA (Schweizerischer Verband Medizinischer PraxisAssistentinnen), www.sva.ch

Diese Regelung soll eine ungerechtfertigte Bevorzugung von Teilzeitangestellten vermeiden Arbeitet eine Teilzeitangestellte über die vereinbarte Teilarbeitszeit hin aus bis zur betrieblichen Arbeitszeit ihrer vollbeschäftigten Kolleginnen, hat sie allenfalls Anspruch auf einen Lohnzuschlag, während ihre Kolleginnen nur den normalen Lohn erhalten.
Wir empfehlen für die Arbeitszeiterfassung, dass die Arbeitszeit mit dem Eintreffen am Arbeitsplatz und mit der Aufnahme der tatsächlichen Arbeitsvorbereitung beginnt (z.B. Computer starten, Wartezimmer lüften oder Zeitschriften bereitlegen, ggf. Post abholen, usw.), und nicht erst mit der völligen Bereitschaft der Arbeitnehmerin am Arbeitsplatz für den Empfang des ersten Patienten / der ersten Patientin. Ebenso gehören auch Arbeiten am Abend, welche zum Praxisbetrieb gehören (z.B. Aufräumen, Arbeitsinstrumente reinigen, usw..), zur Arbeitszeit. Der Arbeitsweg gehört nicht zur Arbeitszeit (vgl. die Ausnahme beim Pikettdienst Ziffer 6.6 des Mustervertrages). Es liegt im Ermessen des Arbeitgebers, mit Erlass von Weisungen die speditive Aufnahme der Arbeit sicherzustellen. Es gibt verschiedene Arbeitszeitmodelle. Neben der im Mustervertrag empfohlenen wöchentlichen Arbeitszeit ist auch eine Jahresarbeitszeitregelung denkbar. Mit der Jahresarbeitszeit wird der Spielraum für die Gestaltung der Arbeitszeit ausgeweitet. Jedoch ist auch hier die maximale Grenze von 50 Stunden pro Woche zu berücksichtigen. Dies sollte jedoch nicht die Regel sein. Grössere Abweichungen vom üblichen Wochenstundenplan bedürfen zudem der Absprache beider Parteien. Idealerweise sollten die Zeitkonti der Arbeitnehmerinnen am Jahresende ausgeglichen sein. Zum Jahresende bestehende Überstundenguthaben sollten aber spätestens bis zur Mitte des Folgejahres durch Freizeitbezug ausgeglichen werden. Dasselbe gilt für Ferienguthaben.

\section{Freie Halbtage (Ziffer 6.2 des Muster- vertrages)}

Gemäss Art. 21 ArG ist der Arbeitnehmerin mindestens jede Woche ein freier Halbtag zu gewähren, wenn die wöchentliche Arbeitszeit auf mehr als fünf Tage verteilt wird. Dies gilt als Mindestanspruch.

\section{Stillende Mütter (Ziffer 6.7 des Muster- vertrages)}

Stillenden Müttern ist die erforderliche Zeit zum Stillen freizugeben und der Arbeitgeber muss, wenn im Betrieb gestillt wird, einen geeigneten Ort zur Verfügung stellen (Art. 34 ArGV 3). Stillenden Müttern muss bei einer täglichen Arbeitszeit von bis zu 4 Stunden 30 Minuten Stillzeit an die bezahlte Arbeitszeit angerechnet werden, bei einer täglichen Arbeitszeit von mehr als 4 Stunden 60 Minuten und bei einer täglichen $\mathrm{Ar}-$ beitszeit von mehr als 7 Stunden 90 Minuten. Es handelt sich bei diesen Bestimmungen nur um Mindestzeiten, die an die bezahlte Arbeitszeit anzurechnen sind. Die Arbeitnehmerin verfügt unabhängig davon, ob sie im Betrieb stillt oder zum Stillen den Arbeitsplatz verlässt, über dieselbe bezahlte Stillzeit. Verlässt sie den Arbeitsplatz zum Stillen, ist für den Weg keine Verlängerung der bezahlten Stillzeit vorgesehen. Anderslautende Abmachungen zwischen dem Arbeitgeber und der betroffenen Arbeitnehmerin sind jedoch möglich.

\section{Gesundheitsschutz bei Mutterschaft (Ziffer 6.9 des Mustervertrages)}

Grundsätzlich hat ein Betrieb mit gefährlichen oder beschwerlichen Arbeiten ${ }^{2}$ eine Risikobeurteilung vorzunehmen. Darin muss insbesondere festgehalten 
sein, welche Gefahren für eine schwangere Frau und stillende Mutter bestehen, wie diese Risiken vermieden werden können und welche Arbeiten während der Schwangerschaft und Stillzeit verboten sind. Der Arbeitgeber hat die Pflicht, die betroffene Mitarbeiterin über die Ergebnisse der Risikobeurteilung zu informieren. Kann eine gefährliche gesundheitliche Belastung für Mutter und Kind nur durch das Ergreifen geeigneter Schutzmassnahmen ausgeschaltet werden, ist deren Wirksamkeit periodisch, mindestens vierteljährlich zu überprüfen.

Eine Vorlage einer Risikobeurteilung des Arbeitsplatzes finden Sie online bei der FMH, Rubrik Mutterschutz in der Arztpraxis, www.fmh.ch/services/medizini sche_praxisassistentin/mutterschutz.html.

\section{Lohnfortzahlung bei Schwangerschaft und Niederkunft (Ziffer $8.1 \mathrm{ff}$. des Mustervertrages)}

Es sind zwei Phasen zu unterscheiden: Die Schwangerschaft, bei der sich die Lohnfortzahlung nach Art. 324a des Schweizerischen Obligationenrechts (OR) richtet, und die Zeit nach der Niederkunft, bei der sich die Ansprüche nach dem Erwerbsersatzgesetz (EOG) richten. Vgl. die Ergänzung zur gesetzlichen Lösung in Ziffer 8.3 Abs. 2 des Mustervertrages.

Der Anspruch auf Mutterschaftsentschädigung beginnt am Tag der Niederkunft und endet spätestens nach 14 Wochen bzw. 98 Tagen. Bei längerem Spitalaufenthalt des Kindes kann die Mutter beantragen, dass der Anspruch auf Entschädigung erst mit der Heimkehr des Kindes beginnt. Die Mutterschaftsentschädigung wird als Taggeld über die AHV-Ausgleichskasse ausgerichtet und beträgt $80 \%$ des vor der Niederkunft erzielten durchschnittlichen Erwerbseinkommens, höchstens aber 196 Franken im Tag.

Hat der Arbeitgeber eine Krankentaggeldversicherung abgeschlossen, kann die Arbeitnehmerin bei Krankheit bei der Versicherung die Differenz zwischen den erwähnten 196 Franken und dem Entschädigungsanspruch der Versicherung (80 Prozent des versicherten Lohns) geltend machen. Und zwar für die Zeit der Erkrankung, aber maximal bis zum Ablauf des Mutterschaftsurlaubs. Sollte die Arbeitnehmerin auch nach Ablauf des Mutterschaftsurlaubs noch krank sein, hat sie Anspruch auf das gesamte Taggeld vom Taggeldversicherer.

Solange eine Mutterschaftsentschädigung ausbezahlt wird, kann kein Taggeld der Arbeitslosenversicherung, der IV oder der obligatorischen Unfallversicherung be- zogen werden. Krankentaggelder der Krankentaggeldversicherung und der obligatorischen Unfallversicherung sind nicht AHV-beitragspflichtig, hingegen sind Erwerbsersatzleistungen gemäss EOG AHV-beitragspflichtig.

\section{Arbeitsverträge und weitere Anstellungs- bedingungen}

Wir empfehlen nachdrücklich die schriftliche Vertragsform auf dem von der FMH und den Berufsverbänden der Medizinischen Praxisassistentinnen gemeinsam erarbeiteten Vertragsformular mit zugehörigen kantonalen Empfehlungen; Bezugsquellen

- Verbindung der Schweizer Ärztinnen und Ärzte FMH, Elfenstrasse 18, Postfach 300, 3000 Bern 15, Tel. 03135911 11, www.fmh.ch, E-Mail: mpa[at]fmh.ch

- Association Romande des Assistantes Médicales ARAM, 1003 Lausanne, Tel. 0793801244 / 079471 68 53, www.aram-vd.ch, E-Mail: info[at]aram-vd.ch

- Schweiz. Verband Medizinischer PraxisAssistentinnen SVA, Postfach 6432, 3001 Bern, Tel. 03138054 54, www.sva.ch, E-Mail: sekretariat[at]sva.ch

Die Berufsverbände der Medizinischen Praxisassistentinnen in Genf und im Tessin haben eine eigene Arbeitsvertragskonvention resp. einen eigenen Arbeitsvertrag; Bezugsquellen:

- Assoziazione Ticinese Assistenti di studio Medico (ATAM), c/o M. Carrera, Via dei Faggi 2 A, 6912 LuganoPazzallo, www.atam.ch, E-Mail: atam[at]bluewin.ch

- Association genevoise des assistantes médicales (AGAM), 1200 Genève, www.agam-ge.ch, E-Mail: presidente[at]agam-ge.ch

\section{Die Löhne für Lernende richten sich nach den kantonalen Empfehlungen.}

FMH-Delegierte für MPA-Fragen:

Dr. med. Adrian Sury, Präsident, Dr. med. Renato Tognina, Vizepräsident

Association genevoise des assistantes médicales (AGAM): Régine Frossard, Präsidentin Assoziazione Ticinese Assistenti di studio Medico (ATAM): Mary Carrera, Sekretariat

Association Romande des Assistantes Médicales ARAM Marie-Paule Fauchère, Präsidentin

Schweizerischer Verband Medizinischer PraxisAssis tentinnen SVA:

Fürsprecher Bruno Gutknecht, Zentralsekretär 